\title{
Selective anticancer effects and protection from chemotherapy by the botanical compound LCS101: Implications for cancer treatment
}

\author{
ZOYA COHEN $^{1 *}$, YAIR MAIMON $^{1 *}$, MERAV YOELI-LERNER $^{1}$, \\ PEIYING YANG ${ }^{2}$, NOAH SAMUELS ${ }^{1}$ and RAANAN BERGER ${ }^{3}$ \\ ${ }^{1}$ Tal Center for Integrative Medicine, Institute of Oncology, Sheba Medical Center, Tel Hashomer, Israel; \\ ${ }^{2}$ Department of General Oncology, The University of Texas M.D. Anderson Cancer Center, \\ Houston, TX, USA; ${ }^{3}$ Institute of Oncology, Sheba Medical Center, Tel Hashomer, Israel
}

Received August 10, 2014; Accepted September 25, 2014

DOI: $10.3892 /$ ijo.2014.2711

\begin{abstract}
There is a need for new options for reducing the side effects of cancer treatment, without compromising efficacy, enabling patients to complete treatment regimens. The botanical compound LCS101 exhibits inhibitory effects on cancer cell growth, and reduces chemotherapy-induced hematological toxicities. The aim of the present study is to examine the selectivity of the effects of the compound, alone and in conjunction with conventional chemotherapy agents, on cancer cell proliferation. The effects of LCS101 were tested on a number of cancer cell lines (breast, MCF7, MDA-MB-231; colorectal, HCT116; prostate, PC-3, DU-145) and on non-tumorigenic normal human epithelial cells (breast, MCF10A; prostate, EP\#2). Cell viability was analyzed using an XTT assay and observed by light microscopy. Necrosis and apoptosis were examined using FACS analysis and immunoblotting. LCS101 selectively induced cell death in breast, colon and prostate cancer cell lines, as measured by XTT assay. Light microscopy and FACS analysis showed changes indicative of a necrotic process. LCS101 was also found to induce PARP-1 reduction in breast cancer cells, with no effect on non-tumorigenic breast epithelial cells. While LCS101 increased cell death in cancer cells exposed to doxorubicin and 5-FU, it showed a protective effect on non-tumorigenic human epithelial cells from chemotherapy-induced cell death. A similar selective effect was observed with apoptosis-associated PARP-1 cleavage. The findings demonstrate that the anti-proliferative effects
\end{abstract}

Correspondence to: Dr Yair Maimon, Tal Center for Integrative Medicine, Institute of Oncology, Chaim Sheba Medical Center, Tel Hashomer 52621, Israel

E-mail: yair@tcm.org.il

*Contributed equally

Key words: cancer, chemotherapy, botanical compounds, cytotoxicity, necrosis, PARP-1, protective exhibited by the botanical compound LCS101 are selective to cancer cells, and offer protection to non-tumorigenic normal epithelial cells from chemotherapy agents.

\section{Introduction}

Cancer is a leading cause of death worldwide (1). Current conventional anticancer treatments incorporate the use of chemotherapy, targeted therapies and radiation treatment. The utilization of these treatments is often limited by severe adverse effects, which often lead to dose reductions and treatment delays. It is therefore important to search for therapies which can reduce the side effects of anticancer treatments without altering their efficacy or increasing toxicity. Such therapies would not only improve the quality of life of patients with cancer but would also help patients complete their anticancer regimen (2).

LCS101 is a botanical compound developed for the treatment of patients with solid cancers, based on the principles of traditional Chinese medicine (TCM). The formula contains concentrated extracts from the following herbs: Astragalus membranaceus, Atractylodes macrocephala, Citrus reticulate, Glehnia littoralis, Ligustrum lucidum, Lycium chinense, Milletia reticulata, Oldenlandia diffusa, Ophiopogon japonicus, Paeonia lactiflora, Paeonia obovata, Poriae cocos, Prunella vulgaris and Scutellaria barbata. Extracts of these compound are manufactured in accordance with good manufacturing practice (GMP) conditions, and are imported under license (Zen Herbs Inc., Rehovot, Israel), in accordance with the regulations of the Israel Ministry of Health. All batches of the compound are analyzed and certified to be free of heavy metals, microbial contamination, pesticide residues and mycotoxins. The herbal components of LCS101 are considered to be safe for human consumption, and have not been found to alter the bioavailability or efficacy of anticancer drugs (3).

In earlier preclinical research, LCS101 was shown in vitro to inhibit breast cancer cell survival in a dose-dependent manner on human breast carcinoma cell lines MDA-231, MDA-453 and T47D. The compound has also shown dosedependent inhibition of cell growth (T47D cell line), as well as 
a dose-dependent increase in cell apoptosis, as demonstrated by sub-diploid DNA content (4). In vivo studies have shown that LCS101 increases peripheral neutrophil counts in doxorubicin-treated mice with breast cancer, preserving splenic erythrocyte and leucocyte counts (unpublished data). LCS101 has also been shown to have indirect anticancer effects, with immunomodulating effects which include the promotion of T-cell proliferation, NK cell activation and cytokine (TNF $\alpha$ ) activity, as well as the correction of reduced IFN- $\gamma$ following exposure to doxorubicin (5). In clinical studies, LCS101 was shown to reduce anemia and neutropenia in female patients with locally advanced breast cancer undergoing anthracycline and taxane-based treatments. In clinical practice and research LCS101 was found to be both safe and well-tolerated by patients (6).

The purpose of the present study was to examine the selectivity of the anticancer effects of LCS101 on cancer cells, and to investigate its impact on the anticancer activity of the chemotherapeutic agents doxorubicin and 5-fluorouracil.

\section{Materials and methods}

Antibodies and reagents. Primary antibodies: rabbit antihuman PARP-1 (polyclonal, 1:1,000) and rabbit anti-human caspase-3 (monoclonal, 1:1,000) were from Cell Signaling Technologies (Boston, MA, USA). Mouse anti-human $\alpha$-tubulin (monoclonal, 1:1,000) was from Sigma-Aldrich (St. Louis, MO, USA). Goat anti-human actin (polyclonal, 1:1,000) and mouse anti-human GAPDH (monoclonal, 1:100,000) were from Santa Cruz Biotechnology (Dallas, TX, USA). Secondary antibodies: peroxidase-conjugated goat anti-rabbit, goat anti-mouse and rabbit anti-goat IgG $(\mathrm{H}+\mathrm{L})$ antibodies were from Jackson (Baltimore Pike West Grove, PA, USA). Propidium iodide, MG132 (Z-Leu-Leu-Leu-al), insulin, doxorubicin, 5-fluorouracil, cholera toxin and hydrocortisone were from Sigma-Aldrich. DMEM F/12, high glucose DMEM, L-glutamine, donor horse serum, fetal bovine serum, recombinant human EGF, trypsin and PBS were from Biological Industries (Beit-Ha-Emek, Israel). Prostate epithelial growth medium was from Lonza (Walkersville, MD, USA).

Cell culture. Human MCF10A non-tumorigenic breast epithelial cells were propagated in DMEM F/12 medium supplemented with $5 \%$ horse serum, $2 \mathrm{ng} / \mathrm{ml}$ epidermal growth factor, $100 \mathrm{ng} / \mathrm{ml}$ cholera toxin, $50 \mathrm{ng} / \mathrm{ml}$ hydrocortisone and $10 \mu \mathrm{g} / \mathrm{ml}$ insulin. Human HCT116 colorectal carcinoma, PC-3 prostate adenocarcinoma, DU-145 prostate carcinoma, MCF7 breast adenocarcinoma and MDA-MB-231 breast adenocarcinoma cells were propagated in high glucose DMEM supplemented with $10 \%$ fetal bovine serum and $2 \mathrm{mM}$ L-glutamine. All cell lines were from American Type Tissue Collection (ATCC, USA) and were authenticated using STR analysis. Human EP\#2 non-tumorigenic normal prostate epithelial cells were donated by Dr Orit Leshem (7) and propagated in prostate epithelial growth medium. All cells were propagated in a $37^{\circ} \mathrm{C}$ humidified incubator with $5 \% \mathrm{CO}_{2}$.

Study compound. A dry extract powder of the formula (Zen Herbs Inc.) was dissolved in PBS at a concentration of $100 \mathrm{mg} / \mathrm{ml}$. The solution was then centrifuged at $4300 \mathrm{~g}$ for
5 min, with the supernatant filtered through a $0.45-\mu \mathrm{M}$ Millex PVDF filter (Merck Millipore, Tullagreen, Ireland). Solubility was estimated by cryophilization and weighting of the pellet, and was estimated to be $\sim 50 \%$. For convenience, the final stock concentration was designated at $100 \mathrm{mg} / \mathrm{ml}$ (w/v concentration of crude powder in PBS), enabling the comparison of the individual herbal components with their variable solubilities with the formula in its entirety.

XTT viability assay. Breast, prostate and colorectal cells were plated in triplicate into $96-$ well plates $(\mathrm{MCF} 10 \mathrm{~A}$ at $6 \times 10^{3} / \mathrm{w}$; MCF7, DU-145 and HCT116 at 3x10 $3 / \mathrm{w}$; PC-3 and

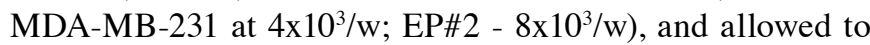
attach and grow overnight. The medium was replaced with a fresh treatment-containing medium, and the cells were propagated for an additional $72 \mathrm{~h}$. Cell viability was determined by XTT cell proliferation kit (Biological Industries) by replacing the medium with a fresh medium (in order to prevent interference of treatment color with XTT signal), and the addition of an XTT for 2-3 h. The resulting signal was measured by Power Wave X 340-I ELISA reader (Biotek Instruments, Winooski, VT, USA), with each cell line tested in at least three independent assays.

FACS analysis. Both cancer and non-tumorigenic cells were plated at a density of $0.6-1 \times 10^{6} / 10 \mathrm{~cm}$ plate, and then treated the following day. The cells were collected by trypsinization into their own medium to prevent loss of dead cells, with each sample divided into two aliquots. The first aliquot was analyzed for necrosis following exposure to a free propidium iodide (PI) influx for $15 \mathrm{~min}$. The second aliquot was fixed with $70 \%$ ice-cold ethanol, stained with PI and used for cell cycle and apoptosis analysis. Cell sorting was performed on a BD FACSCalibur flow cytometer (BD Biosciences, San Jose, CA, USA). Cells were resolved on an FL-2 logarithmic scale for necrosis analysis and on an FL-2 linear scale for apoptosis (cell cycle) analysis, and later analyzed using a WinMDI 2.9 program (Purdue University Cytometry Laboratories, West Lafayette, IN, USA).

Immunoblotting. Cancer and non-tumorigenic cells were plated at a density of $6 \times 10^{5} / 10 \mathrm{~cm}$ plate. On the following day cells were exposed to LCS101 treatments as indicated in the legends. After 24-72 h, the cells were collected by scraping, washed with cold PBS and lysed with RIPA $(150 \mathrm{mM} \mathrm{NaCl}$, $1 \%$ NP-40, 0.5\% deoxycholic acid, $0.1 \%$ SDS, $0.5 \mathrm{M}$ Tris $\mathrm{pH}$ 8.0), supplemented with complete mini protease inhibitor cocktail (Roche Diagnostics GmbH, Mannheim, Germany). Protein concentration was determined with Pierce BCA protein assay kit (Thermo Scientific, Rockford, IL, USA). Samples $(50 \mu \mathrm{g})$ were resolved by $8 \%$ SDS-PAGE, transferred to Protran BA-83 $0.2 \mu \mathrm{m}$ nitrocellulose membrane (Whatman, Piscataway, NJ, USA), blocked with 5\% skimmed milk and immunoblotted with appropriate antibodies. The membrane was then washed thrice with TBST, incubated with corresponding HRP-conjugated secondary antibodies, probed with EZ-ECL enhanced chemiluminescence detection kit (Biological Industries) according to the manufacturer's instructions and then exposed to Fuji Super RX film (Fujifilm, Tokyo, Japan). 


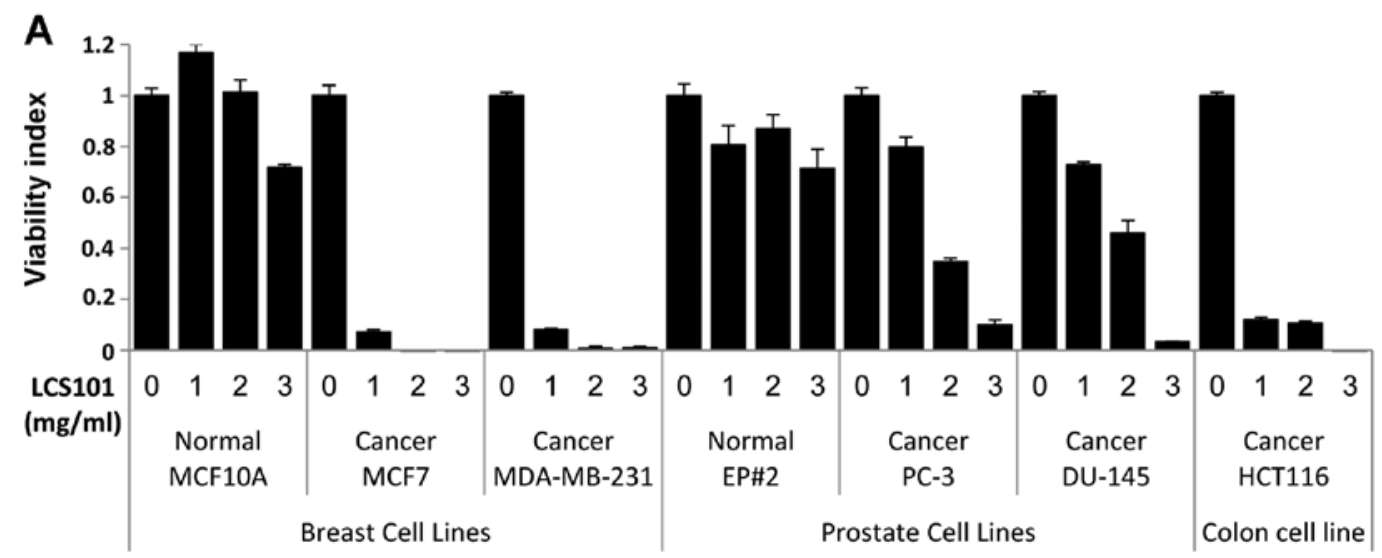

B
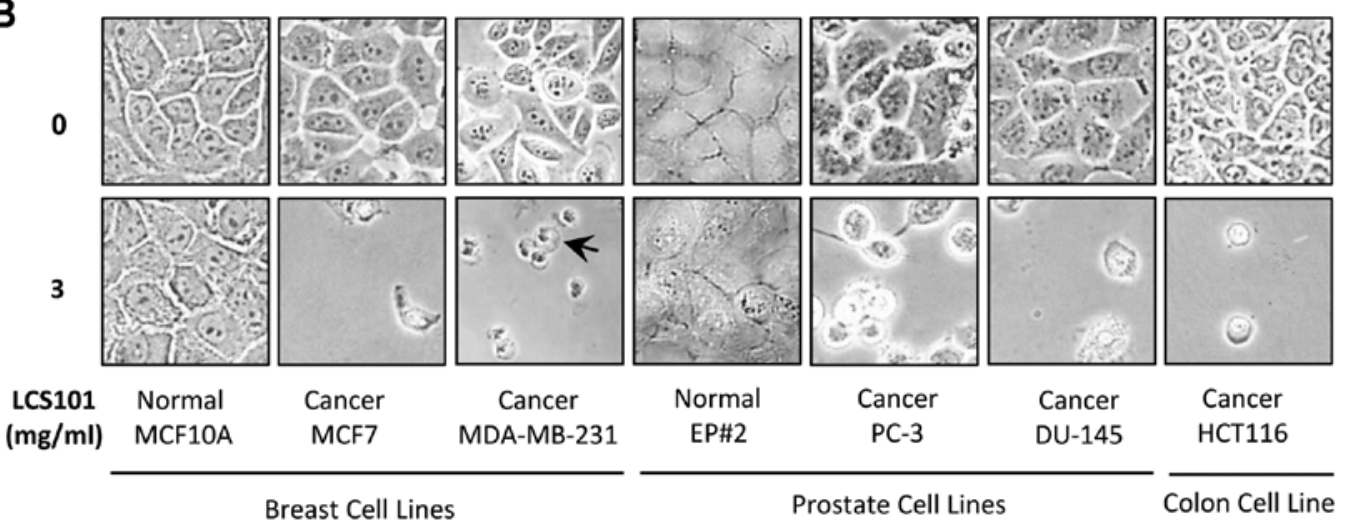

Norma

Cancer

Cancer

Cancer

Prostate Cell Lines

HCT116

Colon Cell Line

Figure 1. LCS101 selectively induces cell death in cancer cells. Non-tumorigenic and cancer cells were treated with incremental concentrations of LCS101. After incubation (72 h) the viability of cells was examined by XTT viability assay (A) and by light microscopy (B). The black arrow in the lower panel of (B) points towards cells with swelling. Error bars in (A) represent the standard \pm deviation.

$R T-q P C R$. Breast cancer cells were plated at a density of $6 \times 10^{5} / 10-\mathrm{cm}$ plates and treated the following day with $3 \mathrm{mg} /$ $\mathrm{ml}$ LCS101. After $24 \mathrm{~h}$ the cells were collected by scrapping and washed with cold PBS. Total RNA was isolated using an RNeasy Mini kit (Qiagen, GmbH, Hilden, Germany). RNA concentration and quality were determined by optic density measurement (260, and $280 \mathrm{~nm}$ ). The quality of the samples was further verified by electrophoresis on $1 \%$ agarose gel, stained with ethidium bromide to visualize the $18 \mathrm{~S}$ and $28 \mathrm{~S}$ rRNA bands. Complementary DNA (cDNA) was prepared using random primers and a High Capacity cDNA Reverse Transcription kit (AB Applied Biosystems, Foster City, CA, USA). cDNA was subjected to RT-qPCR on a StepOnePlus Real-Time PCR System using a Power SYBR Green PCR Master Mix (AB Applied Biosystems). The RT-qPCR was performed according to the manufacturer's instructions using the following primer sets: PARP-1 forward, 5'-AAGCTCT ATCGAGTCGAGTACG-3'; reverse, 5-GAAGCTCAGAGA ACCCATCC-3. GADPH forward, 5-TGGACCTCATGG CCCACA-3; reverse, 5-TCAAGGGGTCTACATGGCAA-3. The expression levels of PARP-1 from triplicate reactions was determined by normalization to GAPDH according to the manufacturer's instructions.

Statistical methods. The mean \pm standard deviations were calculated in each experiment, which were performed in triplicate. The data were collated and analyzed in a Microsoft Excel 2007 program.

\section{Results}

LCS101 selectively induces cell death in cancer cells. Initially we treated the different tumor cell lines and nontumorigenic human cell lines with the LCS101 compound. Exposure of the cultured tumor cells to the compound led to a dose-dependent reduction in cell viability, with cell death observed in $>90 \%$ of cells, as measured by XTT assay. This phenomenon was observed at concentrations of $1 \mathrm{mg} / \mathrm{ml}$ for breast and colon cancer cell lines, and at $3 \mathrm{mg} / \mathrm{ml}$ for prostate cancer cell lines. At the same time, the non-tumorigenic human epithelial cell lines MCF10A (breast) and EP\#2 (prostate) demonstrated a reduction in viability of $<30 \%$ following exposure to the botanical compound (Fig. 1A). The non-tumorigenic human luminal breast cell line HB-2 also displayed an attenuated response to LCS101 exposure (not shown). Light microscopy showed increased cell death of all cancer cells following exposure to LCS101 treatment, with some of the treated cancer cells demonstrating swelled morphology indicative of a necrotic process. In contrast, non-tumorigenic human epithelial cells exhibited normal density and morphology following exposure to the compound (Fig. 1B).

LCS101 reduces PARP-1 expression in breast cancer cell lines. To address the mechanisms underlying the anticancer activity of LCS101, MDA-MB-231, MCF7 and MCF10A breast cells were treated with LCS101, and expression of 
A

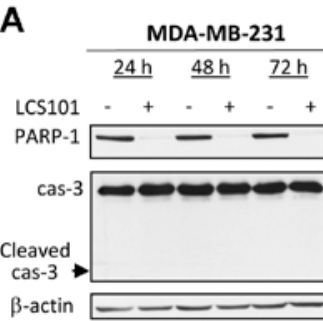

B
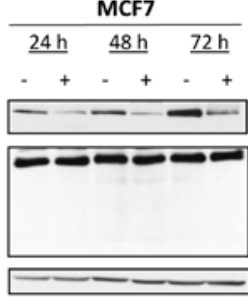

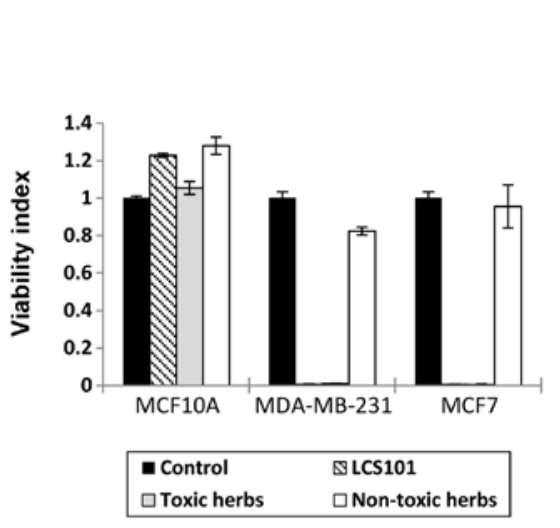

D

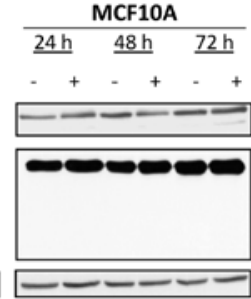

C

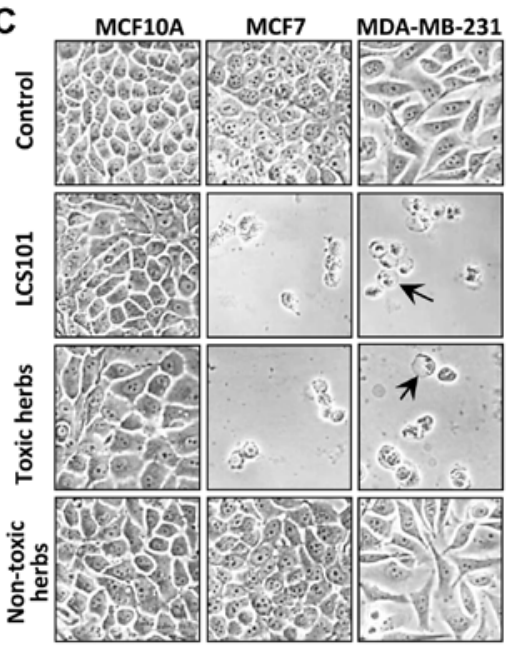

Figure 2. LCS101-induced toxicity correlates with reduction of PARP-1 protein levels. (A) The effect of LCS101 on PARP-1 and caspase-3 (cas-3) in MCF7, MDA-MB-231 and MCF10A cells. (B) Viability of MCF-7, MDA-MB-231 and MCF-10A cells treated with $3 \mathrm{mg} / \mathrm{ml}$ of LCS101 or sub-formulas. XTT viability test was performed at $72 \mathrm{~h}$. Error bars in (A) and (B) represent the standard \pm deviation. (C) Light microscopy. Black arrows in the right panel point towards the MDA-MB-231 cells with swelling. (D) MDA-MB-231 cells, treated as in (B), and subjected to protein extraction and immunoblotting.

apoptosis markers caspase-3 (cas-3) and poly(ADP-ribose) polymerase 1 (PARP-1) were examined. In classic apoptosis both of these proteins undergo cleavage, which is considered as hallmark of apoptosis. Surprisingly, no cleavage of caspase- 3 and PARP-1 was detected, though a significant reduction in the level of PARP-1 protein was observed in both of these cancer cell lines. In contrast, the non-tumorigenic human epithelial breast MCF10A cells exposed to LCS101 showed no reduction in PARP-1 levels (Fig. 2A).

LCS101-induced toxicity correlates with reduced PARP-1 levels. In order to better understand the cytotoxic effects of the LCS101 formulature, 6 of the 14 herbal components were isolated and selected: Ligustrum lucidum, Milletia reticulata, Paeonia lactiflora, Paeonia obovata, Prunella vulgaris and Scutellaria barbata. These herbs were found to display greater toxic effects towards MDA-MB-231 cancer cells, without any harmful effects on the non-tumorigenic human epithelial breast MCF10A cells (not shown). Following these findings, the LCS101 formula was divided into the 'toxic formula' (the above-mentioned 6 components) and the 'nontoxic formula' (the remaining 8 components). Exposure to the toxic formula resulted in a significant increase in cell death in MCF7 and MDA-MB-231 breast cancer lines, compared to no cytotoxic effect in the non-toxic formula (Fig. 2B and C). Following exposure to the toxic formula, cell swelling was observed in treated MDA-MB-231 cells (Fig. 2C, arrows). Both the toxic and non-toxic formulas had
A
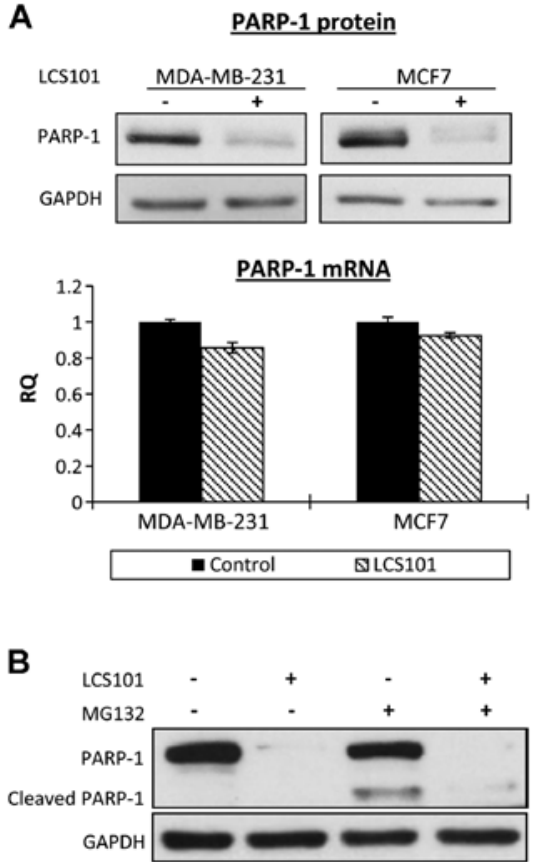

Figure 3. LCS101-induced PARP-1 reduction is unrelated to mRNA or proteasome degradation. (A) MCF7 and MDA-MB-231 cells were plated $6 \times 10^{5}$ cells into $10-\mathrm{cm}$ plates and treated the next day with $3 \mathrm{mg} / \mathrm{ml}$ of LCS101. Protein and RNA were extracted $24 \mathrm{~h}$ after the beginning of the treatment. Protein was immunoblotted with PARP-1 antibody for PARP-1 protein level detection (upper panel). RNA was subjected to RT-qPCR (lower panel). Error bars represent the standard \pm deviation. In both cases GAPDH served as internal control. (B) Western blotting of MCF7 cells treated with $3 \mathrm{mg} / \mathrm{ml}$ of LCS101, $10 \mu \mathrm{M}$ MG132 or both. 


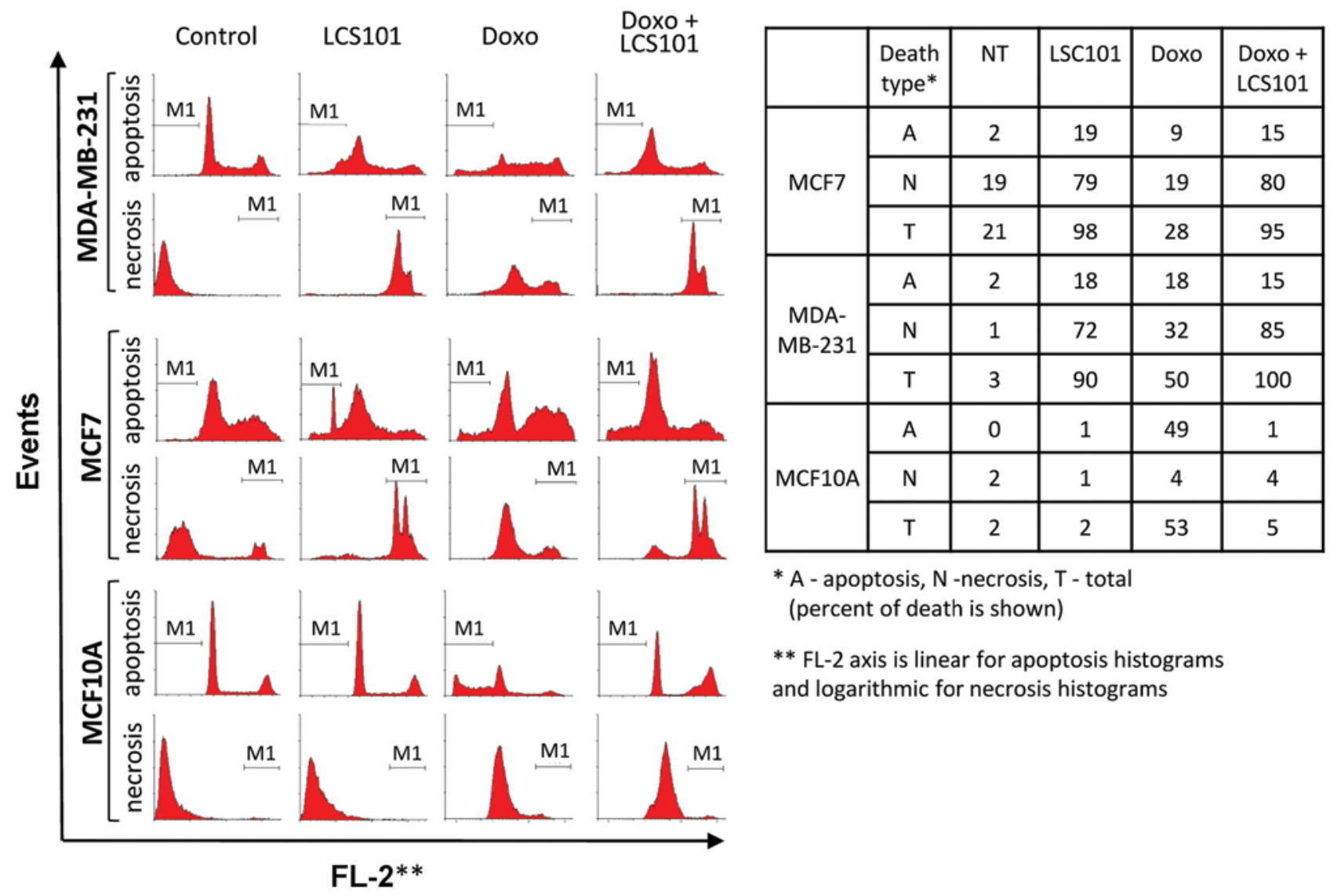

Figure 4. LCS101 protects non-tumorigenic human breast epithelial cells from doxorubicin-induced apoptosis. Cells were plated $6 \times 10^{5} / 10-\mathrm{cm}$ plate and treated the next day with $3 \mathrm{mg} / \mathrm{ml} \mathrm{LCS101}$. After $72 \mathrm{~h}$ of treatment, the cells were collected and each sample was divided to two aliquots. One aliquot was analyzed for necrosis and the second for apoptosis as described in Materials and methods. Cells were resolved on FL-2 logarithmic scale for necrosis analysis and on FL-2 linear scale for apoptosis (cell cycle) analysis. $y$-axis represents percent of cells, $x$-axis - PI intensity. Table shows the percent of cells under the marked area (M1), representing necrotic/apoptotic populations for each cell line and their summary (total).

no effect on the non-tumorigenic human epithelial breast MCF10A cells (Fig. 2B and C). Finally, while the toxic formula reduced PARP-1 levels in MDA-MB-231 cells, no such effect was observed with the non-toxic components of the formula (Fig. 2D).

LCS101-induced PARP-1 reduction is unrelated to mRNA or proteasomal degradation. To further address the mechanism of PARP-1 protein reduction, we tested PARP-1 mRNA level in LCS101-treated cells using RT-PCR. PARP-1 mRNA levels in exposed MCF7 and MDA-MB-231 cells were similar to those found in controls (Fig. 3A). We then examined PARP-1 protein degradation using the proteasome inhibitor MG132 on MDA-MB-231 cells. After $24 \mathrm{~h}$ of treatment with MG132, which was necessary for LCS101-induced PARP-1 reduction, massive cell death and complete PARP-1 cleavage, characteristic of apoptosis, were observed. However, the examination of MCF7 cells, which apparently were more resistant to MG132-induced apoptosis, found that the MG132 failed to prevent PARP-1 elimination following exposure to the botanical formula (Fig. 3B).

LCS101-induced cell death exhibits necrosis-like features. LCS101-treated cells were analyzed using FACS in order to distinguish between apoptotic and necrotic features. For this purpose, MCF7, MDA-MB-231 and MCF10A cells were treated with LCS101 for $72 \mathrm{~h}$, with each sample divided into two aliquots for FACS analysis. One aliquot was fixed and stained with PI to assess the sub-G1 population, which contained cells with degraded DNA, characteristic of apoptotic death. The second aliquot was used to evaluate the percentage of cells with ruptured membranes, typical of necrotic death, using free PI influx by live unfixed cells. Ruptured membrane of necrotic cells allows free PI uptake which causes the necrotic population to appear very bright on logarithmic PI scale. Following exposure to LCS101, the pattern of the vast majority of the cancer cell lines MCF7 and MDA-MB-231 moved far to the right upon free PI uptake, indicating that most of the cells possessed a necrosis-like ruptured membrane (Fig. 4). Morphologically, a number of the affected cells exhibited significant swelling, typical of a necrotic process as well (Fig. 1B). At the same time, only $20 \%$ of the cells exhibited sub-G1 DNA content, a typical indicator of apoptosis (Fig. 4). LCS101 did not induce cell death in the non-tumorigenic human epithelial breast MCF10A cells (Fig. 4).

LCS101 selectively protects non-tumorigenic cells from doxorubicin and 5-FU. Chemotherapy-induced cell death was observed in all three breast cell lines (MCF-10A, MCF-7 and MDA-MB-231) following exposure to the chemotherapy 

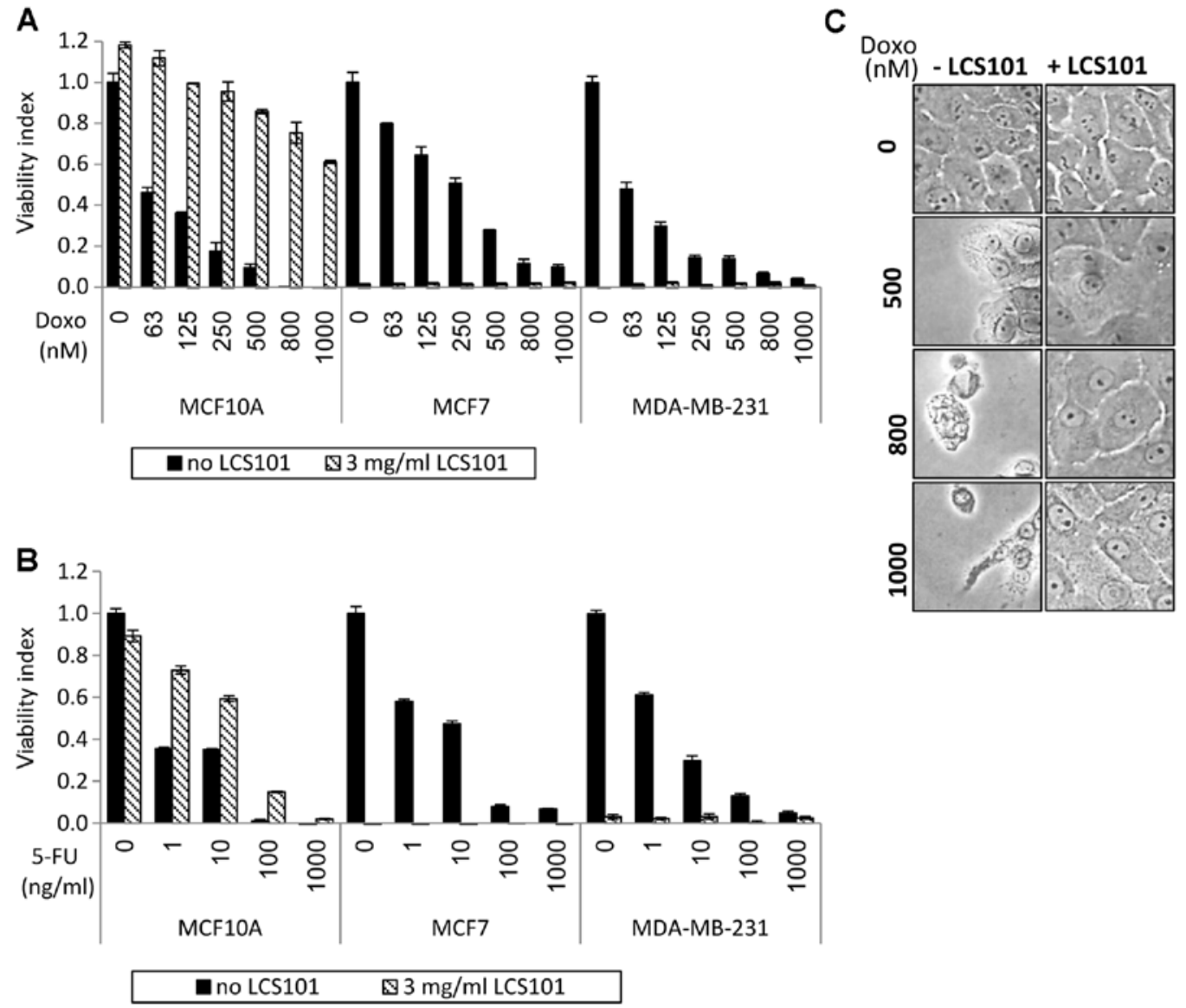

Figure 5. LCS101 selectively protects non-tumorigenic cells from chemotherapy-induced death, without interfering with toxicity to cancer cells. Breast cell lines were treated with rising concentrations of doxorubicin (Doxo) or 5-fluorouracil (5-FU), either alone or in combination with $3 \mathrm{mg} / \mathrm{ml} \mathrm{LCS101.} \mathrm{After} 72 \mathrm{~h}$ of treatment the viability of cells was tested by XTT (A, Doxo; B, 5-FU). Protection of MCF10A cells from doxorubicin-induced death was also examined by light microscopy (C).
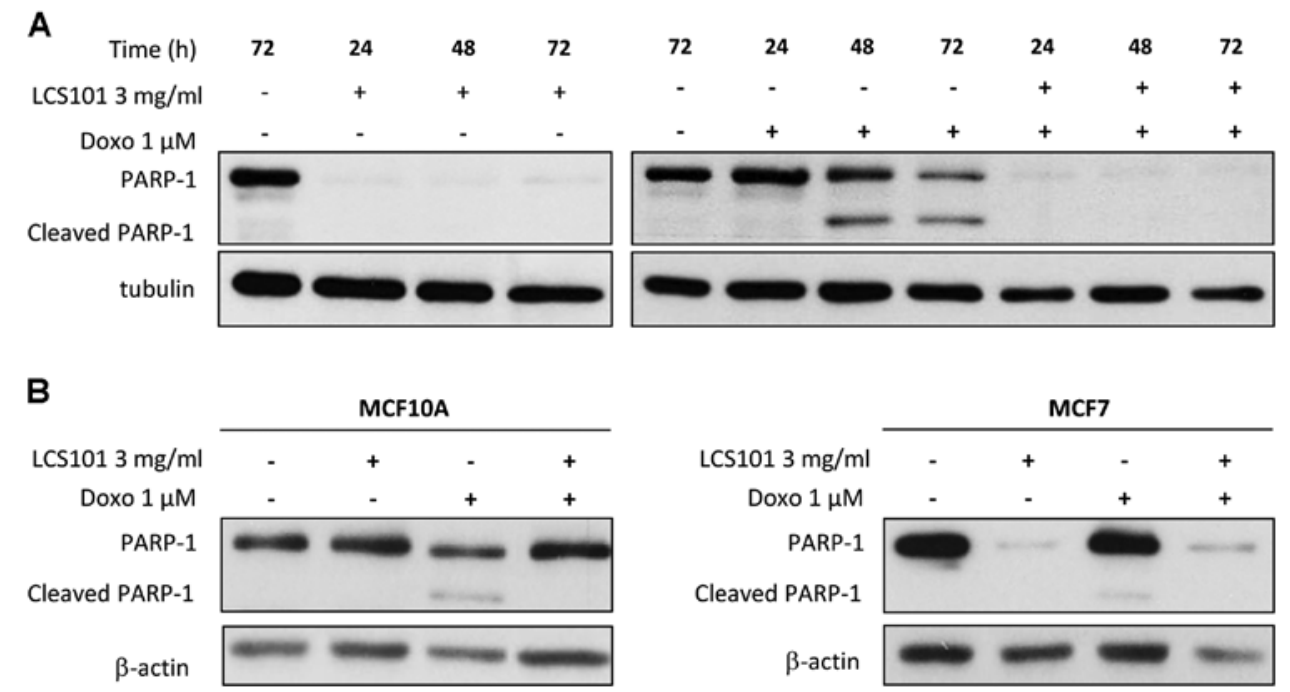

Figure 6. LCS101 selectively reduces PARP-1 levels in doxorubicin-treated cancer cells. MDA-MB-231 (A), MCF10 and MCF7 (B) cells were treated with $3 \mathrm{mg} / \mathrm{ml}$ of LCS101, $1 \mu \mathrm{M}$ doxorubicin or both. Protein was extracted 24-72 $\mathrm{h}$ after the beginning of the treatment for MDA-MB-231 cells and $72 \mathrm{~h}$ after the beginning of the treatment for MCF10A and MCF7 cells, resolved by SDS-PAGE and immunoblotted with PARP-1 antibody and tubulin/ $\beta$-actin for loading control.

agent doxorubicin (Fig. 5A). When introduced at a concentration of $3 \mathrm{mg} / \mathrm{ml}$, LCS101 augmented the tumoricidal effects of the chemotherapy in MCF7 and MDA-MB-231 cells. In contrast, the addition of the botanical formula to the nontumorigenic human epithelial breast MCF10A cells greatly reduced cell death (Fig. 5A and C). A similar selective effect 
Table I. Anticancer effects of LCS101 herbal components.

Herbal component

Anticancer effects

Astragalus membranaceus

Atractylodes macrocephala

Citrus reticulate

Ligustrum lucidum

Oldenlandia diffusa

Paeonia lactiflora

Prunella vulgaris

Scutellaria barbata
Suppression of C6 glioma cells, in vitro and in vivo (9)

Mediation of reactive oxygen species apoptosis in human leukemia cells (10)

Induction of apoptosis in SNU-C4 human colon cancer cells (11)

Induction of apoptosis in human gastric cancer cells (cas-3 pathway) (12)

Induction of human glioma cell death through regulation of Akt/mTOR pathway in vitro and reduction of glioma tumor growth in U87MG xenograph mouse model (13)

Augmentation of oxidative burst in macrophages and inhibited tumor growth (14)

Selective anticancer in vitro effects in B16-F10 mice lung cancer and Renca renal carcinoma models (15)

Inhibition of bladder cancer growth in a rat model involving phosphorylation of Chk2, in vitro and in vivo (16)

Chemoprevention of non-small cell lung cancer (NSCLC) via promotion of apoptosis and regulation of the cell cycle (17)

Suppression of PMA-induced tumor cell invasion and metastasis via inhibition of NF- $\kappa$ B-dependent MMP-9 expression (18)

Induction of oxidative stress damage with redistribution of metabolic fluxes in breast cancer cells (19)

Selective cytotoxic activity on breast cancer cells (20)

Augmentation of oxidative burst in macrophages and inhibited tumor growth (14)

Modulation of apoptosis and cell survival in murine and human prostate cancer cells and tumor development in TRAMP mice (21) was seen in 5-FU-treated cell lines, with LCS101 increasing the tumoricidal effect of the chemotherapy agent on MCF7 and MDA-MB-231 cells, while reducing cell death in the non-tumorigenic human epithelial breast MCF10A cells (Fig. 5B). In addition to the above findings, MDA-MB-231 cells treated with doxorubicin demonstrated PARP-1 cleavage typical of apoptosis, at 48 and at $72 \mathrm{~h}$ (Fig. 6A). A less prominent but still clearly detectable PARP-1 cleavage was observed in MCF10A and MCF7 cells at 72-h treatment with doxorubicin (Fig. 6B). The addition of LCS101 to doxorubicin-treated MDA-MB-231 and MCF7 cells, however, led to the disappearance of PARP-1. In non-tumorigenic human epithelial breast MCF10A cells, LCS101 prevented doxorubicin-induced PARP-1 cleavage altogether, supporting our previous findings showing protective effect of LCS101 in normal cells.

In order to confirm the above findings, we performed FACS analysis of MDA-MB-231, MCF7 and MCF10A cells, which were treated with both doxorubicin and LCS101 (Fig. 4). Our findings were consistent with those above regarding PARP-1 cleavage, with typical apoptosis observed in all three cell lines treated with doxorubicin alone and a clearly demarked sub-G1 apoptotic population. At the same time, the addition of LCS101 reduced apoptosis in nontumorigenic human epithelial breast MCF10A cells, while increasing cell death in the two cancer cell lines (Fig. 4). In cancer cells treated with both LCS101 and doxorubicin, cell death exhibited necrotic features, as described above. This indicates that the increase in doxorubicin-induced cell death in cancer cell lines treated with LCS101 results from a necrosis-like process. At the same time, LCS101 offers a protective effect on non-tumorigenic cells exposed to the chemotherapy agent.

\section{Discussion}

The treatment of patients with cancer presents a number of challenges to oncologists. Anticancer therapies, whether chemotherapy or personalized and targeted biological agents, are often only partially effective and are invariably accompanied by debilitating adverse effects which can compromise the treatment regimen. Many tumors are aggressive and resistant to conventional treatments, which themselves can impair the body's immunity and increase susceptibility to infection. The use of additional chemotherapy agents to established regimens can further increase tumor response, though this positive effect is offset by increased toxicity (8).

Botanical medicine has been in use for thousands of years, with pre-clinical and clinical research demonstrating a number of positive effects of many of the herbal compounds being used for the treatment of cancer, with reduction of disease activity and treatment-related symptoms. In the present study we found the botanical compound LCS101 demonstrated a dose-dependent induction of cell death in 
breast, prostate and colorectal cancer cells. At the same time, LCS101 exhibited no cytotoxic effects on non-tumorigenic human epithelial breast MCF10A cells. The cytotoxic effects of many of the individual LCS101 components have been reported elsewhere in the scientific literature (Table I). Little is known, however, about the selectivity of these effects, and the potential for negative effects on non-tumorigenic cells has limited their use in clinical practice. In light of this, the findings of the present study may have significant implications regarding the incorporation of botanical products into standard anticancer care.

LCS101-induced cancer cell death was manifest as both rupturing of the cell membrane and, in some cases, cell swelling. Both phenomena are demonstrative of a necrotic pathway. The absence of caspase-3 cleavage and lack of DNA degradation despite the massive cell death observed provides further evidence supporting the understanding that this was a manifestation of necrotic cell death. At the same time, however, LCS101-induced cancer cell death was associated with a drastic drop in PARP-1 protein levels, a phenomenon not reported elsewhere in the literature, to the best of our knowledge. This was also observed in the correlation between PARP-1 reduction and cytotoxic effects of the toxic and non-toxic sub-formulas of the botanical compounds. The reduction in PARP-1 levels was not related to either reduction in mRNA expression or proteasomal degradation. Further research is needed in order to understand the implications and mechanisms of these effects on PARP-1 pathways.

We also evaluated the effects of LCS101 on cells treated with the chemotherapy agents doxorubicin and 5-FU. As expected, these agents led to significantly reduced survival in all cell lines. However, while cell death was significantly increased in breast cancer cell lines MCF-7 and MDA-MB-231 following the addition of the botanical compound, nontumorigenic human epithelial breast MCF-10A cells were protected from doxorubicin-induced apoptosis. These findings further support the results observed in earlier clinical trials, in which LCS101 was found to be safe and non-toxic when administered to patients with cancer.

TCM employs a holistic, personalized approach to the treatment of disease. The use of herbal formulas which combine a number of herbal products, each with its own effects on the body acting in harmony with each another, enhances the therapeutic process and promotes well-being. We believe that for this reason, the toxic components need to be supplemented by the non-toxic components in order to promote healing. Many of the LCS101 components have indeed been shown to have anticancer and immunomodulatory affects, as well as demonstrating protective effects against chemotherapy and reactive oxygen species (Table I).

In conclusion, our findings strongly support our previous data suggesting that LCS101 has a cytotoxic effect on cancer cell lines. Furthermore, we show that LCS101 cytotoxicity is selective, with no deleterious effects on non-tumorigenic epithelial cells. LCS101-induced cancer cell death resembles necrosis, though further research is needed in order to better understand this mechanism. In addition, LCS101 provides a selectively protective effect on non-tumorigenic epithelial cells exposed to the chemotherapy agents doxorubicin and 5-FU, while at the same time augmenting their cytotoxic effects on cancer cell lines. Further research is needed to support these findings, as well as understand the clinical implications of this particular botanical compound on anticancer therapy.

\section{Acknowledgments}

Conflict of interest statement: Dr Yair Maimon is a shareholder of LifeBiotics Ltd.

\section{References}

1. World Health Organization fact sheet No. 297; http://www. who.int/mediacentre/factsheets/fs297/en/index.html. Accessed December 28, 2013.

2. Liu SH and Cheng YC: Old formula, new Rx: the journey of PHY906 as cancer adjuvant therapy. J Ethnopharmacol 140: 614-623, 2012.

3. Samuels N, Maimon Y and Zisk-Rony RY: Effect of the botanical compound LCS101 on chemotherapy-induced symptoms in patients with breast cancer: a case series report. Integr Med Insights 8: 1-8, 2013.

4. Maimon Y, Karaush V, Yaal-Hahoshen N, Ben-Yosef R, Ron I, Vexler A and Lev-Ari S: Effect of Chinese herbal therapy on breast cancer adenocarcinoma cell lines. J Int Med Res 38: 2033-2039, 2010

5. Rachmut IH, Samuels N, Melnick SJ, Ramachandran C, Sharabi Y, Pavlovsky A, Maimon Y and Shoham J: Immunomodulatory effects of the botanical compound LCS101: implications for cancer treatment. Onco Targets Ther 6: 437-445, 2013.

6. Yaal-Hahoshen N, Maimon Y, Siegelmann-Danieli N, Lev-Ari S, Ron IG, Sperber F, Samuels N, Shoham J and Merimsky O: A prospective, controlled study of the botanical compound mixture LCS101 for chemotherapy-induced hematological complications in breast cancer. Oncologist 16: 1197-1202, 2011.

7. Leshem O, Madar S, Kogan-Sakin I, Kamer I, Goldstein I, Brosh R, Cohen Y, Jacob-Hirsch J, Ehrlich M, Ben-Sasson S, Goldfinger N, Loewenthal R, Gazit E, Rotter V and Berger R: TMPRSS2/ERG promotes epithelial to mesenchymal transition through the ZEB1/ZEB2 axis in a prostate cancer model. PLoS One 6: e21650, 2011

8. Butters DJ, Ghersi D, Wilcken N, Kirk SJ and Mallon PT: Addition of drug/s to a chemotherapy regimen for metastatic breast cancer. Cochrane Database Syst Rev 2010: CD003368. doi: 10.1002/14651858.CD003368.pub3.

9. Sun JY, Yang H, Miao S, Li JP, Wang SW, Zhu MZ, Xie YH, Wang JB, Liu Z and Yang Q: Suppressive effects of swainsonine on C6 glioma cell in vitro and in vivo. Phytomedicine 16: 1070-1074, 2009.

10. Huang HL, Chen CC, Yeh CY and Huang RL: Reactive oxygen species mediation of baizhu-induced apoptosis in human leukemia cells. J Ethnopharmacol 97: 21-29, 2005.

11. Kang SA, Park HJ, Kim MJ, Lee SY, Han SW and Leem KH: Citri Reticulatae Viride Pericarpium extract induced apoptosis in SNU-C4, human colon cancer cells. J Ethnopharmacol 97: 231-235, 2005.

12. Kim MJ, Park HJ, Hong MS, Park HJ, Kim MS, Leem KH, Kim JB, Kim YJ and Kim HK: Citrus Reticulata blanco induces apoptosis in human gastric cancer cells SNU-668. Nutr Cancer 51: 78-82, 2005.

13. Jeong JC, Kim JW, Kwon CH, Kim TH and Kim YK: Fructus ligustri lucidi extracts induce human glioma cell death through regulation of $\mathrm{Akt} / \mathrm{mTOR}$ pathway in vitro and reduce glioma tumor growth in U87MG xenograft mouse model. Phytother Res 25: 429-434, 2011.

14. Wong BY, Lau BH, Jia TY and Wan CP: Oldenlandia diffusa and Scutellaria barbata augment macrophage oxidative burst and inhibit tumor growth. Cancer Biother Radiopharm 11: 51-56, 1996.

15. Gupta S, Zhang D, Yi J and Shao J: Anticancer activities of Oldenlandia diffusa. J Herb Pharmacother 4: 21-33, 2004.

16. Ou TT, Wu CH, Hsu JD, Chyau CC, Lee HJ and Wang CJ: Paeonia lactiflora Pall inhibits bladder cancer growth involving phosphorylation of Chk2 in vitro and in vivo. J Ethnopharmacol 135: 162-172, 2011 
17. Feng L, Jia X, Zhu M, Chen Y and Shi F: Chemoprevention by Prunella vulgaris L. extract of non-small cell lung cancer via promoting apoptosis and regulating the cell cycle. Asian Pac J Cancer Prev 11: 1355-1358, 2010.

18. Choi JH, Han EH, Hwang YP, Choi JM, Choi CY, Chung YC, Seo JK and Jeong HG: Suppression of PMA-induced tumor cell invasion and metastasis by aqueous extract isolated from Prunella vulgaris via the inhibition of NF-kappaB-dependent MMP-9 expression. Food Chem Toxicol 48: 564-571, 2010.

19. Klawitter J, Klawitter J, Gurshtein J, Corby K, Fong S, Tagliaferri M, Quattrochi L, Cohen I, Shtivelman E and Christians U: Bezielle (BZL101)-induced oxidative stress damage followed by redistribution of metabolic fluxes in breast cancer cells: a combined proteomic and metabolomic study. Int J Cancer 129: 2945-2957, 2011.
20. Fong S, Shoemaker M, Cadaoas J, Lo A, Liao W, Tagliaferri M, Cohen I and Shtivelman E: Molecular mechanisms underlying selective cytotoxic activity of BZL101, an extract of Scutellaria barbata, towards breast cancer cells. Cancer Biol Ther 7: 577-586, 2008.

21. Wong BY, Nguyen DL, Lin T, Wong HH, Cavalcante A, Greenberg NM, Hausted RP and Zheng J: Chinese medicinal herb Scutellaria barbata modulates apoptosis and cell survival in murine and human prostate cancer cells and tumor development in TRAMP mice. Eur J Cancer Prev 18: 331-341, 2009. 